\title{
Special Issue: Language Ideologies and Teaching in Multilingual Contexts
}

\section{Editors:}

Sara Young \& Emma Brooks

Institute of Education, University College London, UK

Text received 4 May 2017, accepted 6 May 2017, final version 29 May 2017

DOI: http://dx.doi.org/10.5565/rev/jt13.728

\section{Notes de les editores \\ Notas de las editoras \\ Editors' notes \\ Lettre des éditrices}

\section{Notes de les editores}

Aquesta monografia recopila articles que tracten sobre les ideologies lingüístiques i com es pot observar l'impacte que tenen sobre l'ensenyament del llengües. En el context del creixent nacionalisme i debats polaritzats sobre la immigració, aquest volum explora com les ideologies presents en la planificació, polítiques i implementació de l'ensenyament del llenguatge influeixen en el disseny curricular, les pràctiques a les aules, i la percepció de la identitat. Clau en tots els articles és el predomini de l'anglès, una llengua amarat amb nocions de prestigi, i aparentment, promogut com una 'moneda' lingüística globalitzada, essencial per accedir a oportunitats futures.

La nostra autora convidada per a aquest volum és la investigadora Andrea Young, qui estudia les ideologies lingüístiques que es presenten en l'ensenyament del francès en un context educatiu. Pel que sembla, tot i les realitats multilingües de les classes de francès actuals, l'ideal monolingüe encara preval.

Nocions de monolingüisme també són prevalents en el segon article, en el qual Clare Courtney destaca l'aparent manca d'importància posada en ESOL (anglès per a parlants d'altres llengües) al Regne Unit. Ella reflexiona a la inconsistència entre els discursos dominants (governamentals i socials) i el finançament del govern, i els efectes d'aquests en les actituds de les i els professors. Allunyant-nos del tema de les ideologies monolingües, Alicia Fernández Barrera documenta el ràpid increment de programes bilingües en escoles d'Espanya central, on l'apropiació de l'anglès permet a les institucions oferir-se en el mercat com a col·legis d'elit i sentir-se orgullosos dins de les comunitats lingüístiques locals 'imaginades' (Anderson, 1991). 
El concepte de comunitats imaginades també és central en l'estudi de Yuta Mogi d'un nihonjingakko, una escola Japonesa complementària a Bèlgica: mentre que l'objectiu de l'escola és ensenyar japonès i cultura als nens vivint en la diàspora, s'observa més èmfasi en la importància d'aprendre anglès com a llengua addicional que a aprendre la llengua local, la francès. La noció de la priorització del global sobre el local es reitera en l'article final, on Joanna Duggan presenta un estudi sobre la selecció de llengües d'ensenyament oficial en les escoles a les Illes Balears. Tot i la coexistència de dues llengües oficials, català i espanyol, hi ha hagut una tendència cap a incrementar l'ensenyament de l'anglès com a llengua estrangera. Després d'aquests articles, John O'Regan li parla a Alexantra Georgiou sobre les ideologies lingüístiques i el predomini global de l'anglès. Aquests temes també són explorats en el llibre que destaquem: Foreign Language Education in Japan: Exploring Qualitative Approache, escrit per Sachiko Horoiguchi, Yuki Imoto, i Gregory S. Poole, ressenya feta per Takako Yoshida.

En resum, els articles en aquesta edició especial examinen les ideologies lingüístiques i els seus efectes en les pràctiques educatives en diversos contextos. Les i els autors van ser contactats per contribuir al volum ja que totes i tots tenen experiència treballant en el camp de l'ensenyament del llenguatge. Esperem que els articles contribueixin al debat actual sobre el paper de l'anglès en l'educació en el sempre-canviant ambient multilingüe.

Sara Young i Emma Brooks, maig-juny 2017

\section{Referència:}

Anderson, B. (Rev Ed) (1991). Imagined communities. Reflections on the origin and spread of nationalism. London: Verso.

\section{Crèdits a:}

Traducció de la nota de les editores i alguns resums: Luis Carabantes (University College London), Sara Young (University College London), Keiko Yuyama (Tamagawa University). Ajuda addicional: Amparo García Ramón, Nicolas Hallet, Glòria Pitarch Ibáñez.

\section{Avaluadors del volum 10.2:}

Les editores volen agrair a les i els següents avaluadors per la seva contribució a la preparació del volum 10.2.

Melanie Cooke (Kings College London), John Gray (University College London, Institute of Education), Vally Lytra (Goldsmiths, University of London), Thomas McAuley (University of Sheffield), Caroline McGlynn (University of East London), Joan Pujolar Cos (Universität Oberta de Catalunya), Raymonde Sneddon (University of East London), Ana Souza (Oxford Brookes University). 


\section{Agraïments}

Els nostres agraïments a Miguel Pérez-Milans i Ana María Relaño-Pastor. Agraïments també a Melinda Dooly per donar-nos l'oportunitat de compilar aquesta monografia, i pel suport que ens ha donat durant aquest procés.

\section{Biodata:}

Sara Young és doctoranda a University College London. La seva recerca explora la construcció d'identitats ètniques i lingüístiques entre adolescents nascuts a Polònia que viuen al Regne Unit. Sara també té un Màster en Investigació de l'Institut d'Educació de University College London; la seva tesi va investigar l'impacte de la crisi econòmica sobra l'ensenyament de l'anglès a Grècia.

\section{Correu clectrònic: s.young.14@ucl.ac.u}

Emma Brooks és doctoranda a University College London. La seva recerca explora les pràctiques comunicatives en consultes prenatals amb dones immigrants. L'estudi se situa en un suburbi súperdiverso de Londres. Emma també treballa com a coordinadora ESOL i està a càrrec d'un projecte sobre les inequitats en salut de les comunitats immigrants.

Correu clectrònic: s.young.14@ucl.ac.u 


\section{Nota de las editoras}

Esta monografía recopila artículos en lo tocante a las ideologías lingüísticas y cómo se observa que éstas impactan la enseñanza del lenguaje. En el contexto del creciente nacionalismo y debates polarizados en inmigración, este volumen explora cómo las ideologías presentes en la planificación, políticas e implementación de la enseñanza del lenguaje influyen en el diseño curricular, las prácticas en la sala de clases, y la percepción de la identidad. Clave en todos los artículos es el predominio del inglés, un idioma empapado con nociones de prestigio, y aparentemente, promovido como una moneda lingüística globalizada, esencial para acceder a oportunidades futuras.

Nuestra autora invitada para este volumen es la investigadora Andrea Young, quien estudia las ideologías lingüísticas que se presentan en la enseñanza del francés en un contexto educacional. Al parecer, a pesar de las realidades multilingües de las clases de francés actuales, el ideal monolingüe aún prevalece.

Nociones de monolingüismo también se destacan en el segundo artículo, donde Clare Courtney recalca la aparente falta de importancia puesta en ESOL (inglés para hablantes de otras lenguas) en el Reino Unido. Ella reflexiona en la inconsistencia entre los discursos dominantes (gubernamentales y sociales) y el financiamiento del gobierno, y los efectos de éstos en las actitudes de las y los profesores. Alejándonos del tema de las ideologías monolingües, Alicia Fernández Barrera documenta el rápido incremento de programas bilingües en escuelas de España central, donde la apropiación del inglés permite a las instituciones ofrecerse en el mercado como colegios de elite y sentirse orgullosos dentro de las comunidades lingüísticas locales ‘imaginadas' (Anderson, 1991).

El concepto de comunidades imaginadas también es central en el estudio de Yuta Mogi de un nihonjingakko, una escuela Japonesa complementaria en Bélgica: mientras que el objetivo de la escuela es enseñar japonés y cultura a los niños viviendo en la diáspora, se observa más énfasis en la importancia de aprender inglés como lengua adicional que en aprender la lengua loca, francés. La idea de la priorización de lo global sobre lo local se reitera en el artículo final, donde Joanna Duggan presenta un estudio sobre la elección de idioma en escuelas en las Islas Baleares. A pesar de la coexistencia de dos lenguas oficiales, catalán y español, ha habido una tendencia hacia incrementar la enseñanza del inglés como lengua extranjera.

Después de estos artículos, John O’Regan le habla a Alexantra Georgiou acerca de las 
ideologías lingüísticas y el predominio global del inglés. Estos temas también son explorados en el libro que destacamos, Foreign Language Education in Japan: Exploring Qualitative Approaches, Sachiko Horoiguchi, Yuki Imoto, y Gregory S. Poole, la reseña está escrita por Takako Yoshida.

Los artículos en esta edición especial examinan las ideologías lingüísticas y sus efectos en las prácticas educativas en varios contextos. Las y los autores fueron invitados a contribuir al volumen ya que todas y todos tienen experiencia trabajando en el campo de la enseñanza del lenguaje. Esperamos que los artículos contribuyan al debate en curso sobre el rol del inglés en la educación en el siempre-cambiante ambiente multilingüe.

Sara Young y Emma Brooks, Mayo-Junio 2017

\section{Referencia:}

Anderson, B. (Rev Ed) (1991). Imagined communities. Reflections on the origin and spread of nationalism. London: Verso.

\section{Créditos a:}

Traducción de las notas de las editores y algunos resúmenes: Luis Carabantes (University College London), Sara Young (University College London), Keiko Yuyama (Tamagawa University). Ayuda adicional: Amparo García Ramón, Nicolas Hallet, Gloria Pitarch Ibáñez.

\section{Evaluadores del volumen $\mathbf{1 0 . 2}$}

Las editoras quisieran agradecer a las y los siguientes evaluadores por su contribución a la preparación del volumen 10.2:

Melanie Cooke (Kings College London), John Gray (University College London, Institute of Education), Vally Lytra (Goldsmiths, University of London), Thomas McAuley (University of Sheffield), Caroline McGlynn (University of East London), Joan Pujolar Cos (Universität Oberta de Catalunya), Raymonde Sneddon (University of East London), Ana Souza (Oxford Brookes University)

\section{Agradecimientos}

Nuestros agradecimientos a Miguel Pérez-Milans y Ana María Relaño-Pastor. Agradecimientos también a Melinda Dooly por darnos la oportunidad de compilar esta monografía, y por el apoyo que nos ha dado durante este proceso.

\footnotetext{
Biodata

Emma Brooks es doctoranda en University College London. Su investigación explora las prácticas comunicativas en consultas prenatales con mujeres inmigrantes. El estudio se sitúa en un suburbio súperdiverso de Londres. Emma también trabaja como coordinadora ESOL y está a cargo de un proyecto acerca de las inequidades en salud de las comunidades inmigrantes.
}

Correo electrónico: e.brooks.14@ucl.ac.uk

Sara Young es doctoranda en University College London. Su investigación explora la construcción de identidades étnicas y lingüísticas entre adolescentes nacidos en Polonia que viven en el Reino Unido. Sara también tiene un Máster en Investigación del Instituto de Educación de University College London; su tesis investigó el impacto de la crisis económica sobra la enseñanza del inglés en Grecia.

Correo clectrónico: $\underline{\text { s.young.14@ucl.ac.u }}$ 


\section{Editors' notes}

This monograph brings together articles on the theme of language ideology and how it may be seen to impact on language teaching. Against the current backdrop of increasing nationalism and polarising debates on migration, this volume explores how ideologies underpinning language planning, policy and implementation influence curriculum design, classroom practice and perception of identity. Key to all articles is the dominance of English, a language imbued with notions of prestige, and seemingly promoted as a linguistic globalised currency, essential for accessing future opportunities.

Our invited author for this volume is the scholar Andrea Young, who examines the language ideologies which underpin teaching in a French educational context. It appears that despite the multilingual realities of contemporary French classrooms, the monolingual ideal still prevails.

Notions of monolingualism are also tackled in the second article, where Clare Courtney highlights the apparent lack of importance attached to ESOL (English for Speakers of Other Languages) in the UK. She reflects on the inconsistency between dominant discourses (governmental and societal) and government funding, and the resulting effects on teacher attitude. Shifting from monolingual ideologies, Alicia Fernández Barrera documents the rapid increase in bilingual programmes in schools in central Spain, where the appropriation of English enables institutions to market themselves as elite and to take pride in local 'imagined' bilingual communities (Anderson, 1991). The concept of imagined communities is also integral to Yuta Mogi's exploration of a nihonjingakko, a Japanese complementary school in Belgium: whilst the aim of the school is to teach Japanese language and culture to children living in the diaspora, there is an emphasis on the importance of learning English as an additional language, rather than the local French. This idea of the prioritisation of the global over the local, is reiterated in the final article, in which Joanna Duggan presents a study of language choice at schools in the Balearic Islands. Despite the coexistence of two official languages, Catalan and Spanish, there has been a move to increase the teaching of English as a foreign language.

Following this, John O'Regan talks to Alexantra Georgiou about language ideology and the global dominance of English. These topics are also explored in our featured book, Foreign Language Education in Japan: Exploring Qualitative Approaches, Sachiko Horoiguchi, Yuki Imoto, and Gregory S. Poole, reviewed by Takako Yoshida.

The papers in this Special Issue thus examine language ideology and its effect on 
teaching practices in various settings. The authors were approached as they all had experience of working in the field of language teaching. We hope the articles in this volume will contribute to the ongoing debate on the role of English in language education in an everchanging multilingual environment.

Sara Young and Emma Brooks May-June 2017

\section{References:}

Anderson, B. (Rev Ed) (1991). Imagined communities. Reflections on the origin and spread of nationalism. London: Verso.

\section{Credits:}

Translations of editorial and some abstracts: Luis Carabantes (University College London), Sara Young (University College London), Keiko Yuyama (Tamagawa University). Additional help: Amparo García Ramón, Nicolas Hallet, Gloria Pitarch Ibáñez.

\section{Reviewers for Volume 10.2}

The editors would like to thank the following reviewers for their contribution to the preparation of Volume 10.2:

Melanie Cooke (Kings College London), John Gray (University College London, Institute of Education), Vally Lytra (Goldsmiths, University of London), Thomas McAuley (University of Sheffield), Caroline McGlynn (University of East London), Joan Pujolar Cos (Universität Oberta de Catalunya), Raymonde Sneddon (University of East London), Ana Souza (Oxford Brookes University)

\section{Acknowledgments}

Our thanks to Miguel Pérez-Milans and Ana María Relaño-Pastor. Many thanks also to Melinda Dooly for giving us the opportunity to compile this monograph, and for the support she has shown throughout the process.

\footnotetext{
Authors' information

Sara Young is a PhD student at University College London. Her doctoral study explores ethnic and linguistic identity construction among Polish-born adolescents living in the UK. Sara also holds an MRes from the UCL Institute of Education; her thesis investigated the impact of the economic crisis on English language teaching in Greece.

Email: s.young.14@ucl.ac.uk
}

Emma Brooks is a PhD student at University College London. Her doctoral research explores communication practices in antenatal consultations with migrant women; the study is situated in a superdiverse London suburb. Emma also works as an ESOL coordinator, and is responsible for an ongoing project addressing health inequalities in migrant communities.

Email: e.brooks.14@ucl.ac.uk 


\section{Lettre des éditrices}

Cette monographie rassemble des articles sur le thème de l'idéologie linguistique et sur son impact perçu sur l'enseignement des langues. Dans le contexte actuel de nationalisme croissant et de débats polarisants sur la migration, ce volume explore comment de telles idéologies, qui sous-tendent la planification linguistique, la politique et la mise en œuvre, influencent la conception de programmes d'études, la pratique en classe et la perception de l'identité. La clé de tous les articles est la domination de l'anglais, une langue imprégnée de notions de prestige, et apparemment promue comme une monnaie linguistique globalisée, indispensable pour accéder aux possibilités futures.

Notre auteur invité pour cet ouvrage est l'académicienne Andrea Young, qui examine les idéologies linguistiques qui sous-tendent l'enseignement dans un contexte éducatif français. Il apparaît que, malgré les réalités multilingues de classes contemporaines françaises, l'idéal monolingue prévaut encore.

Des notions de monolinguisme sont également abordées dans le deuxième article, dans lequel Clare Courtney souligne le manque apparent d'importance accordé à ESOL (anglais pour les locuteurs d'autres langues) au Royaume Uni. Elle réfléchit sur l'incohérence entre les discours dominants (gouvernementaux et sociétaux) et le financement gouvernemental, et les effets qui en résultent sur l'attitude des enseignants. S'éloignant des idéologies monolinguistiques, Alicia Fernández Barrera documente l'augmentation rapide des programmes bilingues dans les écoles du centre de l'Espagne, où l'appropriation permet aux institutions de se présenter comme une élite et d'être fières des communautés bilingues locales « imaginées » (Anderson, 1991). Le concept des communautés imaginées fait aussi partie intégrante de l'exploration par Yuti Mogi d'une nihonjingakko, une école complémentaire japonaise en Belgique: bien que l'objectif de l'école soit d'enseigner la langue et le culture japonaises aux enfants vivant dans la diaspora, on met l'accent sur l'importance d'apprendre l'anglais comme langue supplémentaire, plutôt que le français, la langue locale. Cette idée de la hiérarchisation du global par rapport au local est reprise dans l'étude de Joanna Duggan sur le choix de la langue des écoles aux Iles Baléares. Malgré la coexistence de deux langues officielles, le catalan et l'espagnol, il y a eu un mouvement pour augmenter l'enseignement de l'anglais comme langue étrangère.

Par la suite, John O'Regan parle à Alexantra Georgiou de l'idéologie linguistique et de la domination mondiale de l'anglais. Ces sujets sont également abordés dans notre livre en vedette Foreign Language Education in Japan: Exploring Qualitative Approaches, Sachiko 
Horiguchi, Yuki Imoto, et Gregory S. Poole, examiné par Takako Yoshida.

Les articles de ce Numéro Spécial examinent ainsi l'idéologie linguistique et son effet sur les pratiques pédagogiques dans des divers contextes. Les auteurs ont été approchés puisqu'ils avaient tous une certaine expérience de travail dans le domaine de l'enseignement de langues. Nous espérons que les articles dans ce volume contribueront au débat en cours sur le rôle de l'anglais dans l'enseignement des langues dans un environnement multilingue en constante mutation.

Sara Young et Emma Brooks mai /juin 2017

\section{Référence}

Anderson, B. (Rev Ed) (1991). Imagined communities. Reflections on the origin and spread of nationalism. London: Verso.

\section{Crédits:}

Traductions de la lettre des éditrices et de certains des résumés ont été réalisées par Luis Carabantes (University College London), Sara Young (University College London) et Keiko Yuyama (Tamagawa University). Aide supplémentaire: Amparo García Ramón, Nicolas Hallet, Gloria Pitarch Ibáñez.

\section{Au comité de lecture du volume 10.2}

Les éditrices remercient les personnes suivantes pour leur contribution à la préparation du Volume 10.2:

Melanie Cooke (Kings College London), John Gray (University College London, Institute of Education), Vally Lytra (Goldsmiths, University of London), Thomas McAuley (University of Sheffield), Caroline McGlynn (University of East London), Joan Pujolar Cos (Universitat Oberta de Catalunya), Raymonde Sneddon (University of East London), Ana Souza (Oxford Brookes University).

\section{Remerciements}

Nos remerciements à Miguel Pérez-Milans et Ana María Relaño-Pastor. Nous tenons également à remercier Melinda Dooly pour nous avoir donné l'occasion de compiler cette monographie et pour son constant soutien durant le processus.

\section{Renseignements sur les auteurs}

Sara Young est une doctorante à University College London. Son étude doctorale explore la construction identitaire ethnique et linguistique chez les adolescents nés en Pologne vivant au Royaume-Uni. Sara est également titulaire d'un MRes (Maîtrise en Recherche) de l'Institut d'Education de UCL; sa thèse a étudié l'impact de la crise économique sur l'enseignement d'anglais en Grèce.

Email: s.young.14@ucl.ac.uk

Emma Brooks est une doctorante à University College London. Sa recherche doctorale explore les pratiques de communication dans les consultations prénatales avec les femmes migrantes; l'étude est située dans une banlieue 
« superdiverse » de Londres. Emma travaille également comme une coordinatrice d'ESOL, et est responsable d'un projet en cours sur les inégalités de santé dans les communautés de migrants.

Email: e.brooks.14@ucl.ac.uk

Per citar aquest article/para citar/citer:

Young, S, \& Brooks, E. (2017). Special issue: Language ideologies and teaching in multilingual contexts.

Bellaterra Journal of Teaching \& Learning Language \& Literature, 10(2), 1-10. DOI:

http://dx.doi.org/10.5565/rev/jt13.728

(c) (1) 